\title{
Multi-objective particle swarm optimization with random immigrants
}

\author{
Ali Nadi Ünal ${ }^{1}$ [D $\cdot$ Gülgün Kayakutlu ${ }^{2}$ \\ Received: 25 November 2017 / Accepted: 23 May 2020 / Published online: 12 June 2020 \\ (c) The Author(s) 2020
}

\begin{abstract}
Complex problems of the current business world need new approaches and new computational algorithms for solution. Majority of the issues need analysis from different angles, and hence, multi-objective solutions are more widely used. One of the recently well-accepted computational algorithms is Multi-objective Particle Swarm Optimization (MOPSO). This is an easily implemented and high time performance nature-inspired approach; however, the best solutions are not found for archiving, solution updating, and fast convergence problems faced in certain cases. This study investigates the previously proposed solutions for creating diversity in using MOPSO and proposes using random immigrants approach. Application of the proposed solution is tested in four different sets using Generational Distance, Spacing, Error Ratio, and Run Time performance measures. The achieved results are statistically tested against mutation-based diversity for all four performance metrics. Advantages of this new approach will support the metaheuristic researchers.
\end{abstract}

Keywords Metaheuristics $\cdot$ Multi-objective optimization · Particle swarm optimization $\cdot$ Random immigrants

\section{Introduction}

Nature-inspired optimization methods have been used effectively to solve a wide variety of complex problems that consist of both single and multiple objective search domains. Among these methods, swarm intelligence is a promising research area. Introduced to solve single objective problems, Particle Swarm Optimization (PSO) [15] has attracted many researchers in metaheuristic optimization area, and started to gain prominence at solving multiple objective problems not more than 5 years after its introduction (see [27] for the first attempt on multi-objective optimization). This is because of the relative simplicity and the success as a single-objective optimizer, as well as high speed of convergence [4,22]. Furthermore, due to its population based nature, it enables to obtain a set of trade-off solutions in a single run, unlike the traditional techniques which employ a series of separate runs [36].

Ali Nadi Ünal

anunal@hho.edu.tr

Gülgün Kayakutlu

kayakutlu@itu.edu.tr

1 Hezarfen Aeronautics and Space Technologies Institute, National Defense University, Istanbul, Turkey

2 Energy Institute, Istanbul Technical University, Istanbul, Turkey
However, there still exist three main issues to be considered in Multi-objective Particle Swarm Optimization (MOPSO): (1) archive maintenance, (2) process to update global best and individual best, and (3) solutions for local optima and premature convergence problems $[11,13]$.

Maintaining an external archive, which is used to keep a historical record of non-dominated solutions in accordance with a quality measure, serves the main purpose of multi-objective optimization. Computational cost and memory size considerations cause keeping the size of external archive fixed seems more efficient $[13,29]$. While maintaining the external archive, to obtain a fairly distributed set of non-dominated solutions, employing a density measure in objective space is a straightforward approach. Strategies such as crowding distance [34,37], adaptive grid [5], clustering [33], maximin fitness [21], parallel cell coordinate system [13], and hypersurface contribution [35] can be used for maintaining the archive.

Regarding the update issue, the movement of a particle in MOPSO is affected by personal and global best selection (i.e., the selection of leaders). The selection of leaders is a crucial issue [41], and this selection directly affects the convergence and diversity attitudes, and effectiveness of the algorithm [48]. In other words, the balance between exploitation and exploration capabilities depends on the leader selection process. The trade-off between exploration and exploitation 
is critical to the performance of an evolutionary algorithm [31,47].

Although the fast convergence is an advantage for PSO or MOPSO, it becomes a drawback, if it is not controlled effectively. Fast convergence (premature convergence), especially for the earlier stages of the run, may cause particles to be "trapped in a similar local topology" [9]. As a consequence, it may not be possible to achieve a precise approximation to the true Pareto front [26]. Perturbation operator (mutation, disturbance) is a common practice to compensate for premature convergence, and maintaining diversity of the swarm along the optimization process $[9,13]$.

This paper contributes to the literature by proposing the use of "random immigrants" approach, an effective method to promote diversity for MOPSO. Random immigrant approach has been developed to address maintaining diversity for genetic algorithms and proved to be beneficial [25,43]. It is based on a simple philosophy of replacing the worst or randomly selected particles from the swarm with randomly created particles. To the best of authors' knowledge, ours is the first study to use random immigrants approach for MOPSO.

\section{Literature review}

This section aims to give basic concepts and definitions on multi-objective optimization, and a short survey related to the fast convergence problem mentioned above.

\section{Multi-objective optimization}

Multiple objective optimization problems deal with at least two objective functions to be optimized. These objective functions are non-commensurable and competing. It means that they may be represented in different units, and they may have same level of importance comparatively. Assuming all the objective functions to be minimized, a multi-objective optimization problem can be defined as in [36]:

Minimize $f(\mathbf{x}):=\left[f_{1}(\mathbf{x}), f_{2}(\mathbf{x}), \ldots, f_{k}(\mathbf{x})\right]$

Subject to

$$
\begin{aligned}
& g_{i}(\mathbf{x}) \leq 0 \quad i=1,2, \ldots, m, \\
& h_{j}(\mathbf{x})=0 \quad j=1,2, \ldots, p,
\end{aligned}
$$

where $\mathbf{x}=\left[x_{1}, x_{2}, \ldots, x_{n}\right]^{\mathrm{T}}$ represents decision variables vector, $f_{i}: \mathbb{R}^{n} \rightarrow \mathbb{R}, i=1,2, \ldots, k$ gives objective functions, and $g_{i}, h_{j}: \mathbb{R}^{n} \rightarrow \mathbb{R}, i=1,2, \ldots, m, j=$ $1,2, \ldots, p$ represents inequality and equality constraints, respectively. The desired solution is in the form of "trade-off" solutions between objective functions [34]. In other words, an improvement in one of the objective functions causes worsening for at least one of the others.

The most common two approaches to a multi-objective optimization problem are: (1) transforming the problem into a single objective one; (2) obtaining a set of trade-off solutions (preserving the problem as is) [17]. For the first case, simple additive weighting can be used, or all but one objective function can be moved to the constraint set. However, with the weighting method, not all Pareto-optimal solutions can be found for the problems that have non-convex objective or search spaces [3]. Additionally, many different weights may result the same single solution [3], and it can be very difficult to precisely and accurately select the weights [17]. When moving objective functions to the constraint set, it may be difficult to set right-hand side values for objective functions as constraints.

The main goal of a multi-objective optimization algorithm is to identify solutions in Pareto-optimal set $[17,19]$. Yet, all the elements of a Pareto optimal set may not be desirable or achievable [6,36], and the Pareto-optimal set can be infinite, while we have some space and time limitations. Therefore, it is desirable to obtain a set of solutions that represents the Pareto-optimal set as well as possible [17].

Real-world multi-objective optimization problems may be too complex to be solved by exact methods, such as linear programming and gradient search [50]. Population-based metaheuristic algorithms are accepted as effective computational solvers for multi-objective optimization problems. Due to their search capabilities through large spaces using populations, they are able to get some Pareto-optimal solutions in a single optimization run. Additionally, they are not effected by the shape of the Pareto-optimal front. Of these algorithms, MOPSO is a competitive one. Interested reader should refer [36] for a detailed explanation of a general MOPSO algorithm. Our approach omits the mutation phase of general MOPSO algorithm and adds random immigrants step after updating the leaders in an external archive.

\section{Related work}

Fieldsend and Singh [10] introduced a multi-objective algorithm based on the PSO and demonstrated the inclusion of stochastic turbulence variable. Using this new variable in MOPSO, they showed significant performance increases.

Mostaghim and Teich [28] proposed a method, called Sigma method for selecting the best local guide for each particle. They added a turbulence factor to the updated position of each particle in the swarm.

Xiao-hua et al. [42] proposed a modified PSO named Intelligent Particle Swarm Optimization (IPSO). They used a "clonal selection operator" to accelerate the approximation to optimum. One of the elements in this operator is 
called "clonal mutation", which helps to produce a solution set around Pareto optimal solutions.

Sierra and Coello [37] proposed a multi-objective particle swarm optimizer, which is based on Pareto dominance and uses of a crowding factor. They used uniform and non-uniform mutation schemes. In uniform mutation, the variability range allowed for each decision variable is kept constant over iterations, whilst in non-uniform mutation, this variability decreases over time.

Raquel and Naval [34] proposed an MOPSO algorithm which is called MOPSO-CD. They used crowding distance mechanism and mutation operator to maintain diversity. They performed mutation on the entire swarm initially and then rapidly decreased its coverage over time.

Peng and Zhang [32] proposed a decomposition based MOPSO. They applied the polynomia mutation on positions after they are calculated.

Izui et al. [14] proposed a multi-objective optimization method for structural problems based on MOPSO. They applied a mutation operator. In this operator, the probability of mutation decreases as the number of iterations increases, while mutation rate is fixed.

Agrawal et al. [1] proposed an interactive particle swarm metaheuristic for multi-objective optimization. They employed a mutation operator which is defined "self-adaptive mutation". This operator has some variation in probability according to the number of particles in the repository.

Padhye et al. [30] reviewed some proposals for guide selection in MOPSO and compared them with each other in terms of convergence, diversity, and computational times. They made a proposal named "velocity trigger" as a substitute for turbulence operator coupled with a boundary handling method. They reported that the new proposals were found to be effective for higher objective and higher parameter space problems.

Yen and Leong [44] proposed an MOPSO algorithm with dynamic multiple swarms. In the swarm growing strategy, they use uniform mutation operator with the mutation rate equal to one/number of dimensions in decision space.

Al Moubayed et al. [2] proposed an MOPSO algorithm that employs decomposition. Instead of mutation, they used an information exchange method that helps avoiding local optima without a need for applying any genetic operator.

Yen and Leong [45] proposed a constraint MOPSO which adopts a multi-objective constraint handling technique. They applied uniform and Gaussian operators. Uniform mutation encourage exploration and Gaussian mutation promotes exploitation. The frequency of applying the mutation operators depends on the feasibility ratio of the particles' personal best.

Daneshyari and Yen [7] proposed a cultural MOPSO which adapts the parameters of the MOPSO using the knowledge stored in various parts of the belief space. They applied a time-decaying mutation operator. The number of particles that undergo mutation, the range of mutation for each mutated particle, and the dimensions selected for mutation are regulated accordingly.

Mahmoodabadi et al. [23] modified the MOPSO in two stages. The first stage involves combining PSO with convergence and divergence operators. The second stage involves new leader selection method and adaptive elimination method which aims to limit the number of nondominated solutions in the archive. They used the divergence operator as a simple controlled mutation.

Hu and Yen [12] proposed a method for density estimation for selecting leaders and maintaining the archive in MOPSO. They used "Parallel Cell Distance" between a solution and all other solutions in an archive after the archive is mapped from Cartesian Coordinate System into Parallel Cell Coordinate System. To perturb an article, they used Gaussian Mutation.

Leung et al. [20] presented a new algorithm that extends PSO to deal with multi-objective problems. Their first contribution is that the square root distance computation among particles for local best selection, and second is the procedure to update the archive members. They used mutation operator to enhance the exploratory ability of the algorithm.

Fan, Chang, and Chuang [9] proposed a multi-objective particle swarm optimizer which is constructed based on the concept of Pareto dominance taking both the diversified search and empirical movement strategies into account. They used polynomial mutation to maintaining the diversity of the particles along the optimization process.

$\mathrm{Hu}$ and Yen [13] proposed an integrated and adaptive MOPSO based on Parallel Cell Coordinate System (pccsAMOPSO). Their proposal includes a leader group, self-adaptive parameters, and perturbing operator for balancing convergence and diversity. They employed an elitism learning strategy with a Gaussian mutation as the perturbation operator.

Zhu et al. [49] presented a novel archive-guided MOPSO algorithm (AgMOPSO) where the leaders for velocity update are selected from an external archive. They also used an immune-based evolutionary strategy to evolve the external archive. They stated that this kind of updating scheme was verified to promote the convergence speed and keep the diversity.

Han et al. [11] proposed a variant of MOPSO, named Adaptive Gradient Multi-objective Particle Swarm Optimization (AGMOPSO). They used self-adaptive flight parameters mechanism to balance the convergence and diversity. They claimed that the proposed algorithm can find better spread solutions and has faster convergence to the true Paretooptimal front.

Xiang et al. [41] proposed a many objective PSO (MaPSO). They suggested a new leader selection strategy. They kept multiple historical solutions from which the leader is selected

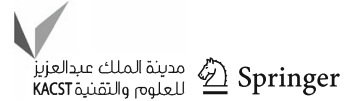


for each particle. They also use linearly decreased parameter which promotes convergence initially and diversity later. It was shown that their proposed MaPSO is highly competitive or significantly superior to other algorithms. In another study on many objective PSO, Luo et al. [22] proposed an algorithm called IDMOPSO. They used a selection strategy for personal best to enhance the capability of local exploration. They also developed a multi-global best selection mechanism to balance convergence and diversity.

Pan et al. [31] proposed a diversity enhanced multiobjective particle swarm optimization called DEMPSO. In that study, analysis of particles' velocities is developed to assist variable clustering and elite selection. A diversity enhancing process based on the velocity analysis is carried out during the particles' evolution.

Current study intents to make a slight extension to [5]. Coello et al. [5] presented an approach in which Paretodominance is incorporated into standard PSO to handle problems with several objective functions. In that study, the movement of a particle is based on its own previous movements (personal best) and the movements of particles in a repository (i.e., leader is selected from an external archive of non-dominated solutions), as well. If the current position of a particle is better than the previous movements, current position is located as personal best. On the other hand, leader is selected randomly from the repository with respect to locations of non-dominated solutions from a hypercube. A more crowded hypercube has less chance to be selected. External archive and the positions of the solutions included in this archive are updated regularly at each iteration. A dynamic mutation operator is also employed in that study. Both the numbers of the particles which are subject to be applied mutation operator and the positions of a particle to be mutated decrease through iterations.

In the current study, we applied the same procedures like [5] except the mutation operator. Instead of mutation operator, random immigrants method is used for diversity preservation.

\section{Random immigrants method}

In the field of evolutionary algorithms, random immigrants method is known as an effective tool for diversity, especially for dynamic optimization $[38,43]$. This method is based on replacing some of the individuals (particles) with new ones. New individuals may be included in a complete random manner, or inclusion may be based on a memory scheme. The predecessors, or omitted individuals, can be selected randomly, or they can be determined with respect to a quality measure (e.g., fitness value or dominance relation).

Coello et al. [5] used mutation operator, such that the number of mutant individuals and the range of effect on decision variables decrease in a nonlinear fashion. In other words, all the particles are affected in the beginning with a high range. As the iteration number gets closer to the end, the number of mutants decreases to almost zero. Doing so, the algorithm gains a highly explorative and leveled (between exploration and exploitation) search capacity.

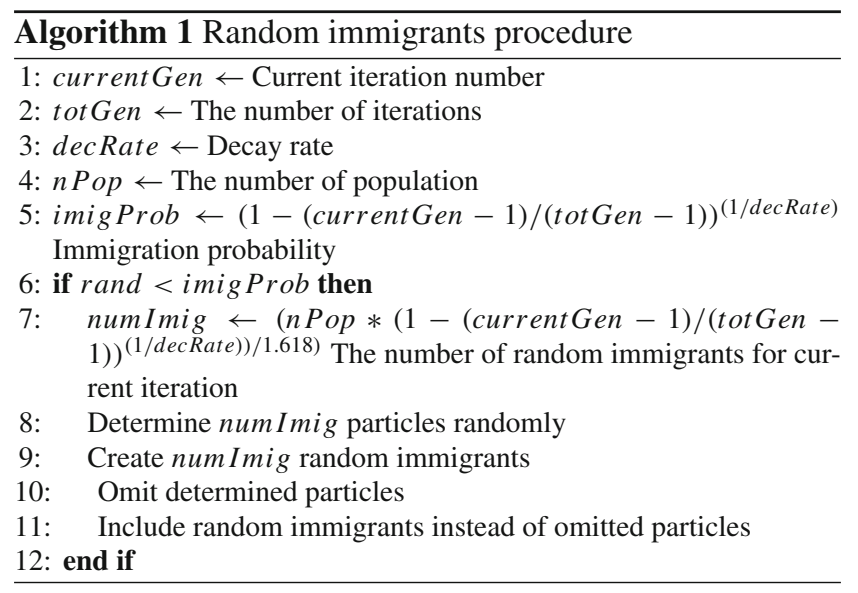

In a previous study [46], Martinez and Coello employed a re-initialization procedure to increase the diversity. In that study, a particle increases its age when it does not improve its personal position, and after exceeding a pre-defined age threshold, the particle is re-initialized. Our approach is totally different by means of re-initialization scheme.

\section{Experimentation}

\section{Test problems}

The first test problem was used by [8], and it is given in Eq. (4):

Minimize $f_{1}\left(x_{1}, x_{2}\right)=x_{1}$
Minimize $f_{2}\left(x_{1}, x_{2}\right)=\frac{g\left(x_{2}\right)}{x_{1}}$

Subject to

$$
\begin{gathered}
g\left(x_{2}\right)=2.0-e^{-\left(\frac{x_{2}-0.2}{0.004}\right)^{2}}-0.8 e^{-\left(\frac{x_{2}-0.6}{0.4}\right)^{2}} \\
0.1 \leq x_{1}, x_{2} \leq 1
\end{gathered}
$$

The second test problem was used by [16], and given in Eq. (5):

Maximize $f_{1}\left(x_{1}, x_{2}\right)=-x_{1}^{2}+x_{2}$
Maximize $f_{2}\left(x_{1}, x_{2}\right)=\frac{1}{2} x_{1}+x_{2}+1$ 
Subject to

$$
\begin{aligned}
& \frac{1}{6} x_{1}+x_{2}-\frac{13}{2} \leq 0 \\
& \frac{1}{2} x_{1}+x_{2}-\frac{15}{2} \leq 0 \\
& \frac{5}{x_{1}}+x_{2}-30 \leq 0 \\
& 0 \leq x_{1}, x_{2} \leq 7
\end{aligned}
$$

The third test problem was used by [18], and it is given in Eq. (6):

Minimize $f_{1}(\mathbf{x})=\sum_{i=1}^{2}-10 e^{-0.2 \sqrt{x_{i}^{2}+x_{i+1}^{2}}}$

Minimize $f_{2}(\mathbf{x})=\sum_{i=1}^{3}\left(\left|x_{i}\right|^{0.8}+5 \sin \left(x_{i}^{3}\right)\right)$

$$
-5 \leq x_{i} \leq 5
$$$$
i=1,2,3
$$

Similar motivation is employed in the current study for random immigrants method. Immigration probability and the number of immigrants decrease with a nonlinear fashion. Random immigrants procedure is shown in Algorithm 1.

The fourth test problem is called "portfolio optimization problem" [24], and it is given in Eq. (7):

$\operatorname{Minimize} \sum_{i=1}^{N} \sum_{j=1}^{N} x_{i} x_{j} \sigma_{i j}$

$\operatorname{Maximize} \sum_{i=1}^{N} x_{i} \mu_{i}$

Subject to

Table 1 Values of parameters

\begin{tabular}{ll}
\hline Parameter & Value \\
\hline Number of iterations & 100 \\
Swarm size & 100 \\
Repository size & 100 \\
$W$ Inertia weight & 0.5 \\
$C_{1}$ Cognitive coefficient & 1 \\
$C_{2}$ Social coefficient & 2 \\
Number of grids & 30 \\
Mutation rate/decay rate & 0.5 \\
Number of runs & 30 \\
\hline
\end{tabular}

$$
\begin{aligned}
& \sum_{i=1}^{N} x_{i}=1, \\
& 0 \leq x_{i} \leq 1, i=1, \ldots, N .
\end{aligned}
$$

The data set for the first three test problems can be obtained from [39], while for the fourth one, it is available in [40].

\section{Performance metrics}

In this study, inspired by the analysis described in [5], three performance metrics are used for comparisons. These metrics are generational distance, spacing, and error ratio, and they are given in Eqs. (8)-(10).

$D=\frac{\sqrt{\sum_{i=1}^{n} d_{i}^{2}}}{n}$,

where $n$ is the number of vectors in the set of nondominated solutions found so far, and $d_{i}$ is the Euclidean distance between each of these and the nearest member of the Pareto optimal set:

$S=\sqrt{\frac{1}{n-1} \sum_{i=1}^{n}\left(\bar{d}-d_{i}\right)^{2}}$,

where $d_{i}=\min _{j}\left(\left|f_{1}^{i}(\mathbf{x})-f_{1}^{j}(\mathbf{x})\right|+\left|f_{2}^{i}(\mathbf{x})-f_{2}^{j}(\mathbf{x})\right|\right), i, j=$ $1, \ldots, n, i \neq j, \bar{d}$ is the mean of all $d_{i}$, and $n$ is the number of nondominated vectors so far:

$E=\frac{\sum_{i=1}^{n} e_{i}}{n}$,

where $n$ is the number of vectors in the current set of nondominated vectors available, $e_{i}=0$ if the vector $i$ is a member of the Pareto optimal set, and $e_{i}=1$, otherwise. In addition to the above-mentioned metrics, run times are also evaluated to compare the speed of the two competing algorithms.

\section{Hypothesis testing and parameters}

To test how effective the proposed approach is, we compared two algorithms, namely MOPSO with mutation and MOPSO with random immigrants. We run both algorithms 30 times for each of the four problems. We compared both algorithms with respect to four performance metrics, both statistically and graphically. Statistical hypothesis testing $(z$ test) is performed for comparison. The null hypothesis and the alternative hypothesis are stated as:

$H_{0}: \mu_{1}=\mu_{2}$ There is no significant difference between two approaches with respect to the related performance metric. 
Fig. 1 Pareto-fronts for the first test problem
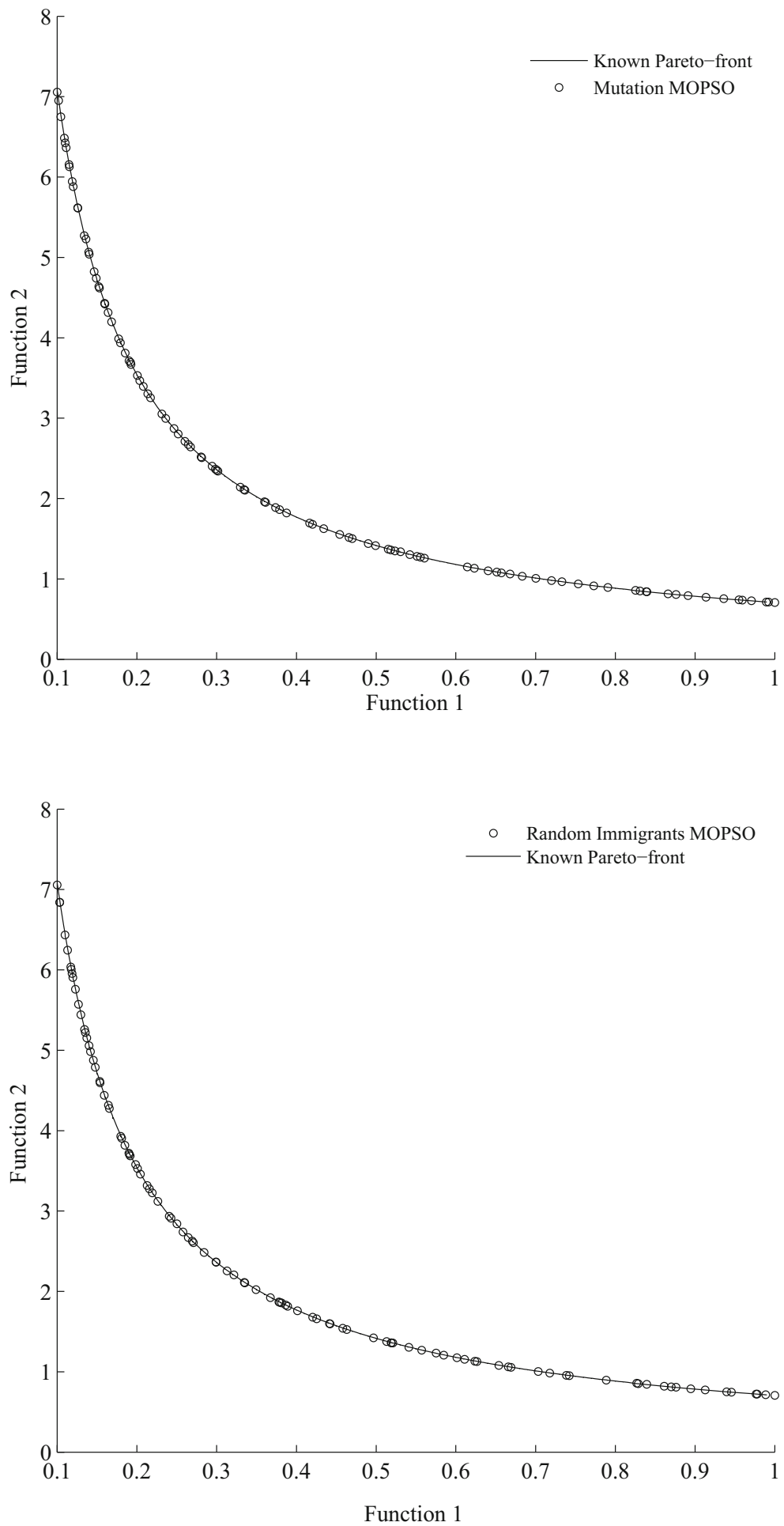

$H_{1}: \mu_{1} \neq \mu_{2}$ There is a significant difference between two approaches with respect to the related performance metric.

Here, $\mu_{1}$ represents a mean value of 30 runs for respective performance metric for mutation MOPSO, and $\mu_{2}$ represents a mean value of 30 runs for respective performance metric for random immigrants MOPSO. The parameters for both approach are given in Table 1. 
Fig. 2 Pareto-fronts for the second test problem
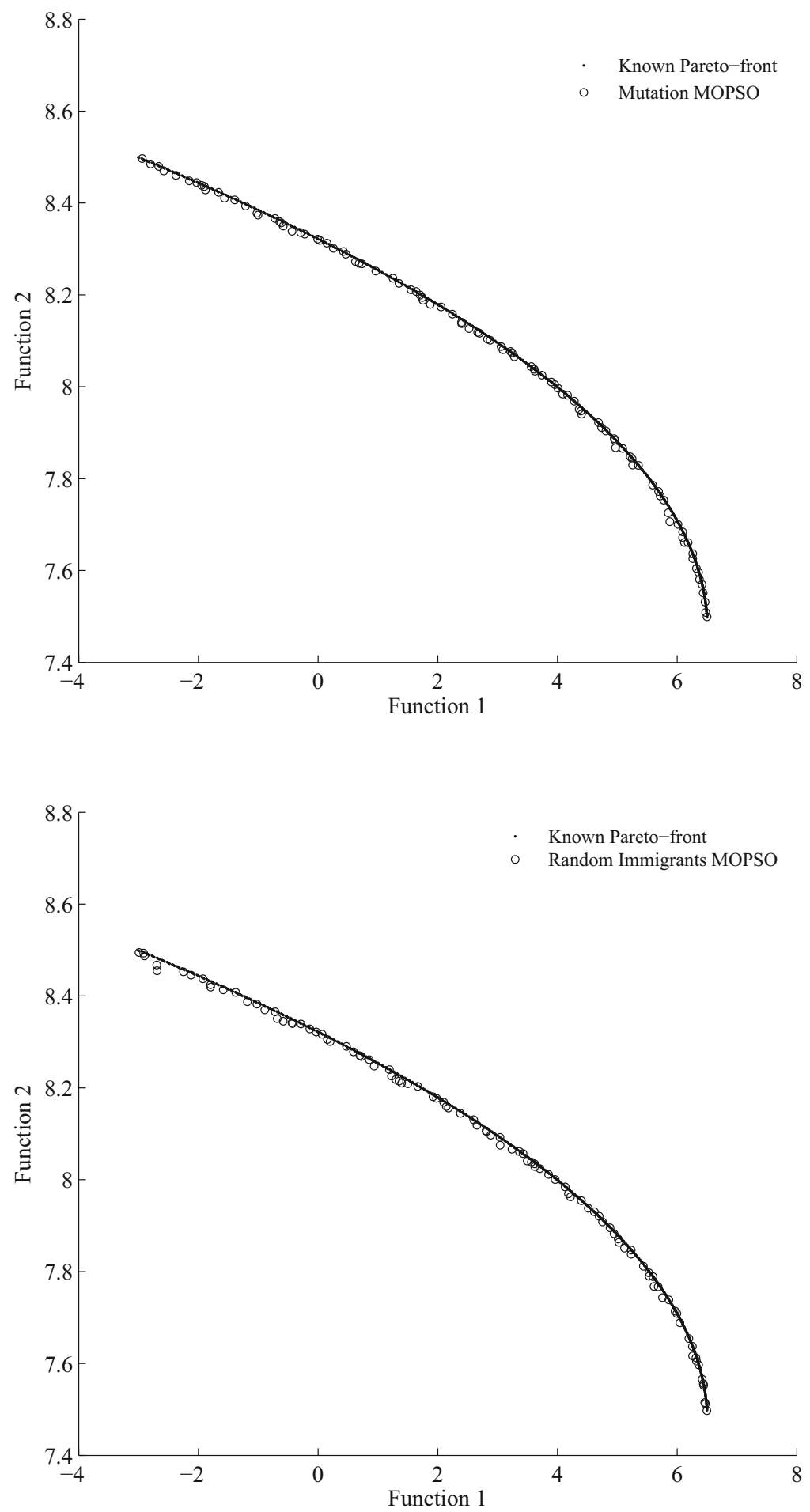

\section{Results}

Graphical comparison for all four test problems is given in Figs. 1, 2, 3 and 4. The results shown in these figures represent the best solutions with respect to the $D$ performance metric. It can be seen that both mutation and random immigrants
MOPSO produce a good approximation for the real Paretofront for given test problems.

For each test problem, results for each run with respect to each performance metric are given in Tables 2, 4, 6, and 8, respectively. Based on these results, z-test is performed, and $z$ values are given in Tables 3, 5, 7, and 9 . 
Fig. 3 Pareto-fronts for the third test problem
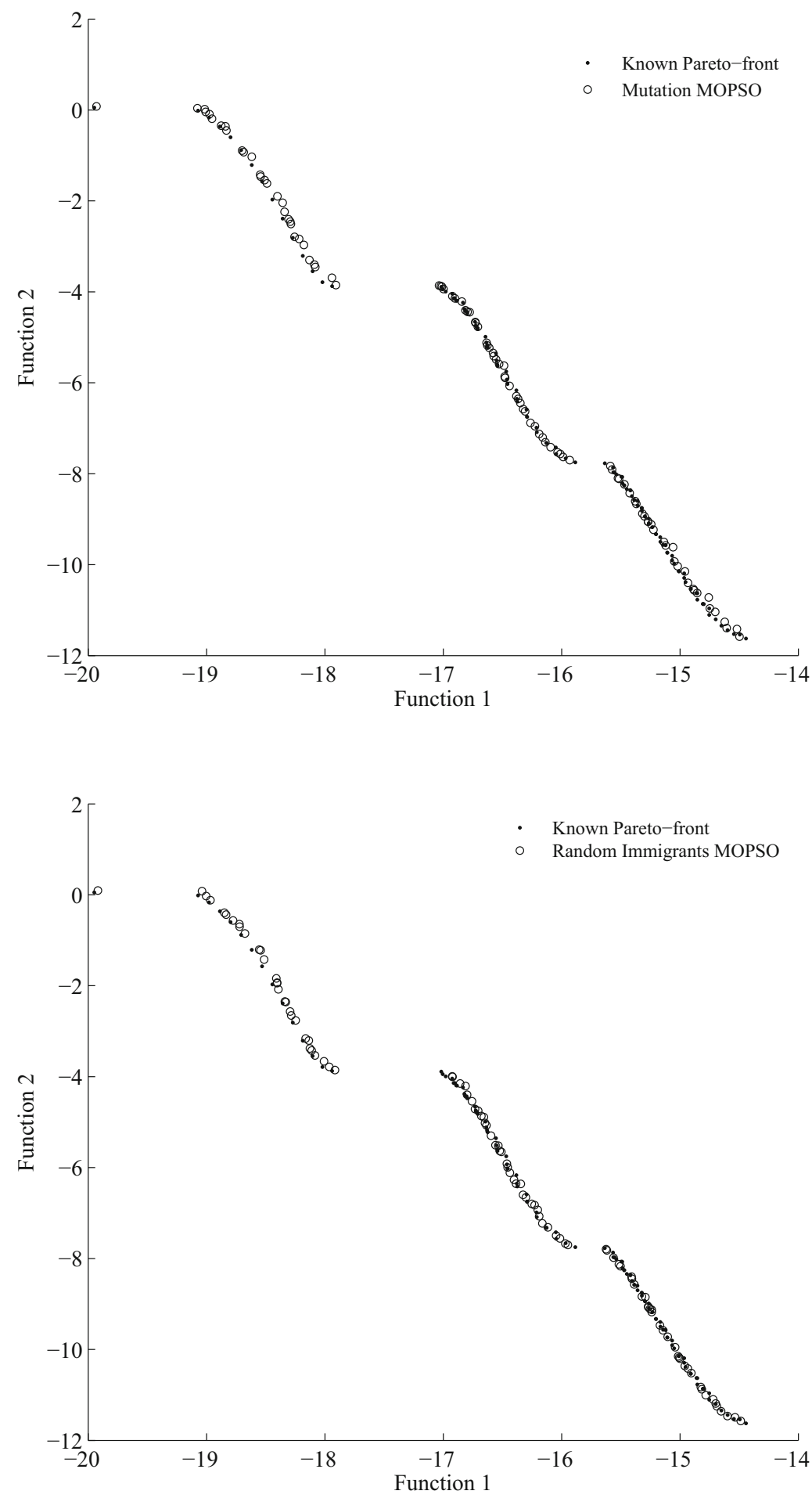

\section{Results for the first test problem}

According to the results given in Tables 2 and 3 for $S$ performance metric null hypothesis can be rejected. It means that there is a significant difference between two approaches, with respect to the mean values of $S$. It seems that the mutation
MOPSO outperforms random immigrants MOPSO for this performance metric.

Regarding the $D$ and $E$ performance metrics, there is not enough evidence to reject null hypothesis. It means that there is no significant difference between two approaches, with respect to the mean values of $D$ and $E$. For the time performance metric, the null hypothesis can be rejected. It means 
Fig. 4 Pareto-fronts for the fourth test problem
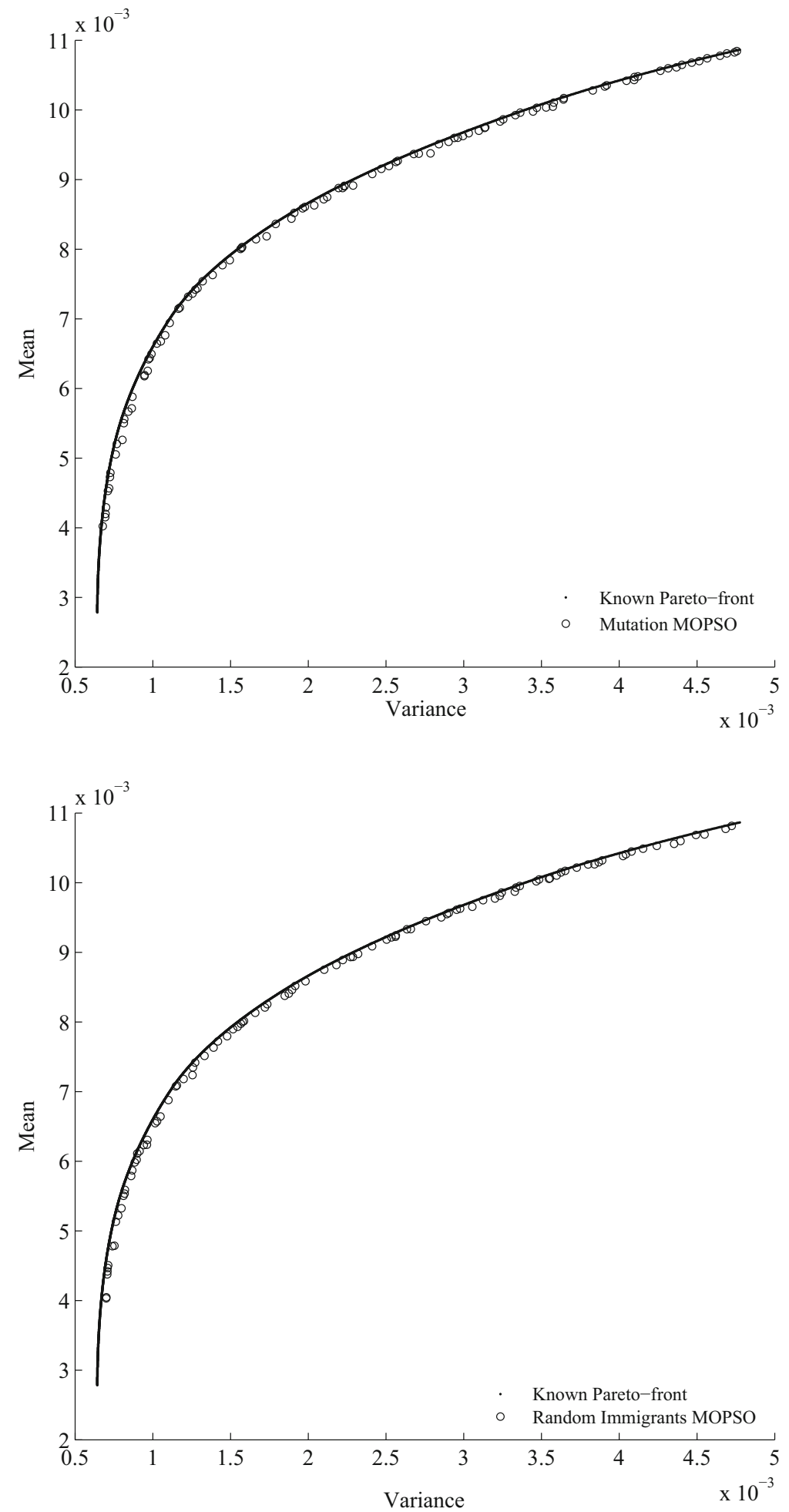

that there is a significant difference between the approaches, with respect to the run times of the algorithms. Random immigrants MOPSO seems to outperform mutation MOPSO method.

\section{Results for the second test problem}

According to the results given in Tables 4 and 5 , for $S, D$, and $E$ performance metrics, null hypothesis cannot be rejected. In other words, there is no significant difference between two approaches. Regarding the time performance metric, null 
Table 2 Results for the first test problem

\begin{tabular}{|c|c|c|c|c|c|c|c|c|}
\hline \multirow[t]{3}{*}{ Run/statistic } & \multicolumn{8}{|c|}{ Performance metrics } \\
\hline & \multicolumn{2}{|l|}{$S$} & \multicolumn{2}{|l|}{$D$} & \multicolumn{2}{|l|}{ E } & \multicolumn{2}{|l|}{$T$} \\
\hline & RI & Mut. & RI & Mut. & RI & Mut. & RI & Mut. \\
\hline 1 & 0.0426 & 0.0435 & 0.0038 & 0.0039 & 0.0200 & 0.0300 & 42.7821 & 46.3837 \\
\hline 2 & 0.0429 & 0.0365 & 0.0043 & 0.0040 & 0.0100 & 0.0400 & 43.7493 & 48.4386 \\
\hline 3 & 0.0401 & 0.0423 & 0.0044 & 0.0048 & 0.0100 & 0.0400 & 44.2675 & 51.7774 \\
\hline 4 & 0.0443 & 0.0326 & 0.0038 & 0.0043 & 0.0200 & 0.0300 & 43.7091 & 48.6544 \\
\hline 5 & 0.0455 & 0.0358 & 0.0046 & 0.0040 & 0.0300 & 0.0300 & 43.2256 & 46.3498 \\
\hline 6 & 0.0374 & 0.0366 & 0.0044 & 0.0048 & 0.0300 & 0.0300 & 43.3359 & 48.6472 \\
\hline 7 & 0.0431 & 0.0384 & 0.0042 & 0.0051 & 0.0100 & 0.0200 & 43.6625 & 48.7798 \\
\hline 8 & 0.0444 & 0.0376 & 0.0044 & 0.0043 & 0.0300 & 0.0100 & 44.6642 & 47.8639 \\
\hline 9 & 0.0436 & 0.0423 & 0.0043 & 0.0040 & 0.0200 & 0.0100 & 43.0536 & 46.9575 \\
\hline 10 & 0.0374 & 0.0404 & 0.0047 & 0.0043 & 0.0400 & 0.0200 & 45.1330 & 48.7515 \\
\hline 11 & 0.0331 & 0.0345 & 0.0048 & 0.0051 & 0.0100 & 0.0300 & 44.7500 & 48.9877 \\
\hline 12 & 0.0346 & 0.0349 & 0.0043 & 0.0040 & 0.0300 & 0.0200 & 44.1840 & 49.5633 \\
\hline 13 & 0.0327 & 0.0391 & 0.0043 & 0.0045 & 0.0300 & 0.0400 & 42.6265 & 47.4781 \\
\hline 14 & 0.0372 & 0.0315 & 0.0040 & 0.0038 & 0.0200 & 0.0200 & 45.2132 & 49.0925 \\
\hline 15 & 0.0432 & 0.0389 & 0.0044 & 0.0042 & 0.0200 & 0.0200 & 44.3408 & 47.6364 \\
\hline 16 & 0.0416 & 0.0363 & 0.0044 & 0.0041 & 0.0200 & 0.0100 & 45.2360 & 50.4313 \\
\hline 17 & 0.0385 & 0.0362 & 0.0044 & 0.0045 & 0.0300 & 0.0400 & 42.2915 & 47.4614 \\
\hline 18 & 0.0357 & 0.0352 & 0.0042 & 0.0044 & 0.0300 & 0.0300 & 44.3946 & 48.3598 \\
\hline 19 & 0.0453 & 0.0431 & 0.0043 & 0.0041 & 0.0300 & 0.0100 & 45.7289 & 46.5018 \\
\hline 20 & 0.0419 & 0.0386 & 0.0046 & 0.0043 & 0.0300 & 0.0200 & 44.4128 & 47.3061 \\
\hline 21 & 0.0433 & 0.0366 & 0.0042 & 0.0046 & 0.0100 & 0.0400 & 42.8689 & 46.8911 \\
\hline 22 & 0.0481 & 0.0331 & 0.0043 & 0.0046 & 0.0300 & 0.0200 & 43.5025 & 47.4834 \\
\hline 23 & 0.0422 & 0.0435 & 0.0045 & 0.0045 & 0.0300 & 0.0000 & 43.0032 & 47.7383 \\
\hline 24 & 0.0385 & 0.0313 & 0.0042 & 0.0041 & 0.0100 & 0.0200 & 46.0204 & 47.9371 \\
\hline 25 & 0.0459 & 0.0396 & 0.0041 & 0.0049 & 0.0500 & 0.0100 & 43.5170 & 48.5421 \\
\hline 26 & 0.0339 & 0.0290 & 0.0047 & 0.0044 & 0.0400 & 0.0300 & 44.2943 & 47.6873 \\
\hline 27 & 0.0416 & 0.0323 & 0.0048 & 0.0043 & 0.0300 & 0.0300 & 44.0153 & 47.5622 \\
\hline 28 & 0.0388 & 0.0409 & 0.0044 & 0.0040 & 0.0400 & 0.0100 & 42.5261 & 47.1278 \\
\hline 29 & 0.0355 & 0.0283 & 0.0044 & 0.0049 & 0.0300 & 0.0100 & 45.7032 & 47.0328 \\
\hline 30 & 0.0390 & 0.0425 & 0.0048 & 0.0052 & 0.0300 & 0.0500 & 44.3875 & 48.6581 \\
\hline Min. & 0.0327 & 0.0283 & 0.0038 & 0.0038 & 0.0100 & 0.0000 & 42.2915 & 46.3498 \\
\hline Max. & 0.0481 & 0.0435 & 0.0048 & 0.0052 & 0.0500 & 0.0500 & 46.0204 & 51.7774 \\
\hline Mean & 0.0404 & 0.0370 & 0.0044 & 0.0044 & 0.0257 & 0.0240 & 44.0200 & 48.0694 \\
\hline Std. dev. & 0.0041 & 0.0043 & 0.0003 & 0.0004 & 0.0104 & 0.0122 & 1.0076 & 1.1910 \\
\hline
\end{tabular}

Table 3 Statistical hypothesis testing for the first test problem

\begin{tabular}{lll}
\hline Performance metric & $z$ value & $H_{0}$ \\
\hline $\mathrm{S}$ & 3.08 & Reject \\
$\mathrm{D}$ & -0.4496 & Not enough evidence to reject \\
$\mathrm{E}$ & 0.5694 & Not enough evidence to reject \\
$\mathrm{T}$ & -14.2174 & Reject \\
\hline$\alpha<0.05$ & &
\end{tabular}


Table 4 Results for the second test problem
Table 5 Statistical hypothesis testing for the second test problem

\begin{tabular}{|c|c|c|c|c|c|c|c|c|}
\hline \multirow[t]{3}{*}{ Run/statistic } & \multicolumn{8}{|c|}{$\underline{\text { Performance metrics }}$} \\
\hline & \multicolumn{2}{|l|}{$\underline{S}$} & \multicolumn{2}{|l|}{$D$} & \multicolumn{2}{|l|}{$E$} & \multicolumn{2}{|l|}{$T$} \\
\hline & RI & Mut. & RI & Mut. & RI & Mut. & RI & Mut. \\
\hline 1 & 0.0491 & 0.0374 & 0.0113 & 0.0139 & 0.1100 & 0.1200 & 38.6895 & 38.1807 \\
\hline 2 & 0.0484 & 0.0515 & 0.0119 & 0.0344 & 0.1800 & 0.1500 & 39.3091 & 37.9143 \\
\hline 3 & 0.0648 & 0.0480 & 0.0176 & 0.0267 & 0.1700 & 0.0900 & 36.5373 & 40.3929 \\
\hline 4 & 0.0539 & 0.0771 & 0.0233 & 0.0142 & 0.1000 & 0.1300 & 37.6547 & 38.1014 \\
\hline 5 & 0.1574 & 0.1580 & 0.0503 & 0.1526 & 0.0900 & 0.1200 & 38.1928 & 38.2802 \\
\hline 6 & 1.5723 & 0.1409 & 0.1738 & 0.0905 & 0.1100 & 0.1100 & 36.6740 & 38.3075 \\
\hline 7 & 0.0515 & 0.9726 & 0.0408 & 0.1995 & 0.1700 & 0.0600 & 37.7982 & 36.8344 \\
\hline 8 & 0.2944 & 0.0542 & 0.0634 & 0.0246 & 0.1300 & 0.0700 & 38.4847 & 54.4839 \\
\hline 9 & 0.0504 & 0.0576 & 0.0084 & 0.0159 & 0.1400 & 0.1900 & 39.9653 & 54.3228 \\
\hline 10 & 0.1311 & 0.0652 & 0.1657 & 0.0404 & 0.1400 & 0.1700 & 34.6981 & 38.9263 \\
\hline 11 & 0.0411 & 0.0811 & 0.0124 & 0.0227 & 0.1200 & 0.0900 & 37.0576 & 36.7245 \\
\hline 12 & 0.1304 & 0.1706 & 0.0361 & 0.0255 & 0.1900 & 0.1500 & 36.5183 & 38.0513 \\
\hline 13 & 0.1097 & 0.3508 & 0.0224 & 0.0768 & 0.2000 & 0.0900 & 37.5736 & 39.4193 \\
\hline 14 & 0.9711 & 0.0460 & 0.1132 & 0.0679 & 0.1500 & 0.1100 & 37.4087 & 39.1830 \\
\hline 15 & 0.0516 & 0.7488 & 0.0218 & 0.0924 & 0.1000 & 0.1300 & 39.1586 & 38.4872 \\
\hline 16 & 0.4443 & 0.1697 & 0.1241 & 0.0271 & 0.1700 & 0.1600 & 37.9423 & 38.1918 \\
\hline 17 & 0.0855 & 0.1534 & 0.0408 & 0.0239 & 0.1200 & 0.1500 & 38.7080 & 39.3768 \\
\hline 18 & 0.0466 & 0.0505 & 0.0231 & 0.0074 & 0.1100 & 0.1100 & 38.2719 & 37.9889 \\
\hline 19 & 0.5780 & 0.7758 & 0.0693 & 0.0940 & 0.0900 & 0.1100 & 37.0737 & 37.6973 \\
\hline 20 & 0.7394 & 0.0577 & 0.2124 & 0.0581 & 0.1500 & 0.1400 & 36.2117 & 38.6942 \\
\hline 21 & 0.0447 & 0.7446 & 0.0094 & 0.1712 & 0.1800 & 0.1800 & 37.2069 & 37.6676 \\
\hline 22 & 0.0603 & 0.1034 & 0.0120 & 0.0245 & 0.1100 & 0.1300 & 37.2697 & 38.9032 \\
\hline 23 & 0.0462 & 0.1047 & 0.0122 & 0.0794 & 0.1900 & 0.1300 & 37.3081 & 39.4277 \\
\hline 24 & 0.2703 & 0.0457 & 0.0463 & 0.0145 & 0.1000 & 0.0900 & 38.5350 & 39.0234 \\
\hline 25 & 0.0545 & 0.0439 & 0.0301 & 0.0166 & 0.1500 & 0.1200 & 37.1355 & 39.7306 \\
\hline 26 & 0.0429 & 0.1315 & 0.0097 & 0.1110 & 0.1000 & 0.1600 & 39.3758 & 36.6069 \\
\hline 27 & 0.0489 & 0.0920 & 0.0109 & 0.0276 & 0.1300 & 0.1700 & 38.8031 & 38.8613 \\
\hline 28 & 0.0829 & 0.0541 & 0.0509 & 0.0087 & 0.1100 & 0.1800 & 38.0753 & 38.8249 \\
\hline 29 & 0.2253 & 0.4364 & 0.0429 & 0.1417 & 0.1400 & 0.0900 & 38.9690 & 37.4339 \\
\hline 30 & 0.0833 & 0.0465 & 0.0232 & 0.0153 & 0.1300 & 0.1200 & 38.9606 & 39.9278 \\
\hline Min. & 0.0411 & 0.0374 & 0.0084 & 0.0074 & 0.0900 & 0.0600 & 34.6981 & 36.6069 \\
\hline Max. & 1.5723 & 0.9726 & 0.2124 & 0.1995 & 0.2000 & 0.1900 & 39.9653 & 54.4839 \\
\hline Mean & 0.2210 & 0.2023 & 0.0497 & 0.0573 & 0.1360 & 0.1273 & 37.8522 & 39.5322 \\
\hline Std. dev. & 0.3408 & 0.2605 & 0.0540 & 0.0530 & 0.0329 & 0.0338 & 1.1341 & 4.1443 \\
\hline
\end{tabular}

\begin{tabular}{lll}
\hline Performance metric & $z$ value & $H_{0}$ \\
\hline $\mathrm{S}$ & 0.2387 & Not enough evidence to reject \\
$\mathrm{D}$ & -0.5539 & Not enough evidence to reject \\
$\mathrm{E}$ & 1.007 & Not enough evidence to reject \\
$\mathrm{T}$ & -2.1514 & Reject \\
\hline$\alpha<0.05$ & &
\end{tabular}


Table 6 Results for the third test problem

Table 7 Statistical hypothesis testing for the third test problem

\begin{tabular}{|c|c|c|c|c|c|c|c|c|}
\hline \multirow[t]{3}{*}{ Run/statistic } & \multicolumn{8}{|c|}{$\underline{\text { Performance metrics }}$} \\
\hline & \multicolumn{2}{|l|}{$S$} & \multicolumn{2}{|l|}{$D$} & \multicolumn{2}{|l|}{$E$} & \multicolumn{2}{|l|}{$\underline{T}$} \\
\hline & RI & Mut. & RI & Mut. & RI & Mut. & RI & Mut. \\
\hline 1 & 0.1086 & 0.1080 & 0.0589 & 0.0558 & 0.0000 & 0.0000 & 30.7471 & 31.9891 \\
\hline 2 & 0.0999 & 0.1021 & 0.0595 & 0.0579 & 0.0000 & 0.0000 & 32.7430 & 31.2913 \\
\hline 3 & 0.0694 & 0.1115 & 0.0590 & 0.0579 & 0.0400 & 0.0000 & 31.3615 & 30.7032 \\
\hline 4 & 0.0887 & 0.1043 & 0.0523 & 0.0549 & 0.0300 & 0.0000 & 31.4082 & 31.5288 \\
\hline 5 & 0.1169 & 0.1165 & 0.0512 & 0.0591 & 0.0000 & 0.0000 & 31.2170 & 29.6907 \\
\hline 6 & 0.0595 & 0.0651 & 0.0561 & 0.0602 & 0.0000 & 0.0000 & 31.2949 & 31.0227 \\
\hline 7 & 0.0735 & 0.0953 & 0.0619 & 0.0519 & 0.0200 & 0.0000 & 31.8885 & 30.3834 \\
\hline 8 & 0.0955 & 0.0577 & 0.0578 & 0.0604 & 0.0000 & 0.0300 & 30.8581 & 31.2546 \\
\hline 9 & 0.1176 & 0.1085 & 0.0579 & 0.0584 & 0.0000 & 0.0100 & 30.4882 & 30.3319 \\
\hline 10 & 0.1047 & 0.0590 & 0.0532 & 0.0563 & 0.0000 & 0.0200 & 31.8226 & 31.3167 \\
\hline 11 & 0.1056 & 0.1011 & 0.0531 & 0.0602 & 0.0000 & 0.0000 & 30.8987 & 30.2592 \\
\hline 12 & 0.1006 & 0.1155 & 0.0554 & 0.0542 & 0.0000 & 0.0000 & 30.8969 & 30.8225 \\
\hline 13 & 0.1017 & 0.0573 & 0.0597 & 0.0570 & 0.0000 & 0.0300 & 30.6360 & 30.0280 \\
\hline 14 & 0.1069 & 0.1062 & 0.0587 & 0.0595 & 0.0000 & 0.0000 & 32.1974 & 30.5088 \\
\hline 15 & 0.0622 & 0.1063 & 0.0568 & 0.0599 & 0.0000 & 0.0000 & 31.1471 & 30.7564 \\
\hline 16 & 0.0952 & 0.1072 & 0.0563 & 0.0528 & 0.0100 & 0.0000 & 31.4530 & 31.2568 \\
\hline 17 & 0.1009 & 0.0987 & 0.0544 & 0.0581 & 0.0000 & 0.0100 & 31.6583 & 31.3700 \\
\hline 18 & 0.0535 & 0.1076 & 0.0569 & 0.0547 & 0.0000 & 0.0000 & 31.9432 & 31.1027 \\
\hline 19 & 0.0668 & 0.0657 & 0.0546 & 0.0583 & 0.0000 & 0.0000 & 30.9934 & 31.1797 \\
\hline 20 & 0.0972 & 0.1110 & 0.0503 & 0.0632 & 0.0000 & 0.0000 & 31.4585 & 30.0472 \\
\hline 21 & 0.1124 & 0.0644 & 0.0601 & 0.0545 & 0.0000 & 0.0000 & 31.6670 & 29.9122 \\
\hline 22 & 0.1111 & 0.0725 & 0.0582 & 0.0598 & 0.0100 & 0.0200 & 31.1748 & 30.2498 \\
\hline 23 & 0.0592 & 0.1118 & 0.0594 & 0.0567 & 0.0200 & 0.0000 & 31.5038 & 31.4885 \\
\hline 24 & 0.0688 & 0.0562 & 0.0568 & 0.0595 & 0.0000 & 0.0100 & 31.5334 & 31.7842 \\
\hline 25 & 0.1021 & 0.1056 & 0.0535 & 0.0558 & 0.0000 & 0.0000 & 31.7340 & 31.4312 \\
\hline 26 & 0.1066 & 0.1073 & 0.0544 & 0.0544 & 0.0000 & 0.0000 & 31.5341 & 30.5024 \\
\hline 27 & 0.0703 & 0.1016 & 0.0542 & 0.0601 & 0.0000 & 0.0100 & 31.6510 & 31.0848 \\
\hline 28 & 0.0917 & 0.0611 & 0.0657 & 0.0593 & 0.0100 & 0.0300 & 31.8327 & 30.6619 \\
\hline 29 & 0.1258 & 0.0951 & 0.0512 & 0.0585 & 0.0000 & 0.0000 & 30.8778 & 31.0640 \\
\hline 30 & 0.1013 & 0.0598 & 0.0557 & 0.0573 & 0.0000 & 0.0000 & 31.4873 & 30.5123 \\
\hline Min. & 0.0535 & 0.0562 & 0.0503 & 0.0519 & 0.0000 & 0.0000 & 30.4882 & 29.6907 \\
\hline Max. & 0.1258 & 0.1165 & 0.0657 & 0.0632 & 0.0400 & 0.0300 & 32.7430 & 31.9891 \\
\hline Mean & 0.0925 & 0.0913 & 0.0564 & 0.0575 & 0.0047 & 0.0057 & 31.4036 & 30.8512 \\
\hline Std. dev. & 0.0202 & 0.0219 & 0.0034 & 0.0026 & 0.0101 & 0.0101 & 0.4895 & 0.5828 \\
\hline
\end{tabular}

\begin{tabular}{lll}
\hline Performance metric & $z$ value & $H_{0}$ \\
\hline $\mathrm{S}$ & 0.2069 & Not enough evidence to reject \\
$\mathrm{D}$ & 1.4085 & Not enough evidence to reject \\
$\mathrm{E}$ & -0.3844 & Not enough evidence to reject \\
$\mathrm{T}$ & 3.9755 & Reject \\
\hline$\alpha<0.05$ & &
\end{tabular}


Table 8 Results for the fourth test problem

\begin{tabular}{|c|c|c|c|c|c|c|c|c|}
\hline \multirow[t]{3}{*}{ Run/statistic } & \multicolumn{8}{|c|}{$\underline{\text { Performance metrics }}$} \\
\hline & \multicolumn{2}{|l|}{$\underline{S}$} & \multicolumn{2}{|l|}{$D$} & \multicolumn{2}{|l|}{$E$} & \multicolumn{2}{|l|}{$T$} \\
\hline & RI & Mut. & RI & Mut. & RI & Mut. & RI & Mut. \\
\hline 1 & $6.22 \mathrm{E}-05$ & $4.72 \mathrm{E}-05$ & $3.62 \mathrm{E}-05$ & $3.77 \mathrm{E}-05$ & 0.44 & 0.35 & 37.449 & 38.873 \\
\hline 2 & $4.48 \mathrm{E}-05$ & $4.23 \mathrm{E}-05$ & $3.90 \mathrm{E}-05$ & $3.7 \mathrm{E}-05$ & 0.4 & 0.29 & 36.834 & 37.131 \\
\hline 3 & $4.24 \mathrm{E}-05$ & $4.48 \mathrm{E}-05$ & $3.18 \mathrm{E}-05$ & $3.13 \mathrm{E}-05$ & 0.33 & 0.35 & 38.478 & 37.947 \\
\hline 4 & $4.14 \mathrm{E}-05$ & $4.37 \mathrm{E}-05$ & $3.49 \mathrm{E}-05$ & $3.63 \mathrm{E}-05$ & 0.38 & 0.32 & 37.497 & 37.047 \\
\hline 5 & $4.21 \mathrm{E}-05$ & $4.41 \mathrm{E}-05$ & $3.39 \mathrm{E}-05$ & $3.84 \mathrm{E}-05$ & 0.41 & 0.33 & 37.722 & 37.162 \\
\hline 6 & $4.75 \mathrm{E}-05$ & $4.51 \mathrm{E}-05$ & $3.55 \mathrm{E}-05$ & $4.13 \mathrm{E}-05$ & 0.33 & 0.34 & 37.098 & 37.436 \\
\hline 7 & $4.26 \mathrm{E}-05$ & $4.06 \mathrm{E}-05$ & $3.53 \mathrm{E}-05$ & $2.69 \mathrm{E}-05$ & 0.32 & 0.35 & 37.250 & 37.927 \\
\hline 8 & $5.03 \mathrm{E}-05$ & $5.47 \mathrm{E}-05$ & $3.28 \mathrm{E}-05$ & $4.5 \mathrm{E}-05$ & 0.35 & 0.29 & 36.809 & 38.579 \\
\hline 9 & $5.1 \mathrm{E}-05$ & $4.34 \mathrm{E}-05$ & $3.97 \mathrm{E}-05$ & $4.3 \mathrm{E}-05$ & 0.39 & 0.31 & 37.178 & 38.249 \\
\hline 10 & $5.33 \mathrm{E}-05$ & $4.21 \mathrm{E}-05$ & $3.55 \mathrm{E}-05$ & $3.37 \mathrm{E}-05$ & 0.34 & 0.39 & 42.377 & 37.440 \\
\hline 11 & $4.35 \mathrm{E}-05$ & $4.96 \mathrm{E}-05$ & $3.09 \mathrm{E}-05$ & $2.84 \mathrm{E}-05$ & 0.41 & 0.37 & 43.261 & 37.812 \\
\hline 12 & $5.7 \mathrm{E}-05$ & $4.71 \mathrm{E}-05$ & $3.94 \mathrm{E}-05$ & $3.31 \mathrm{E}-05$ & 0.24 & 0.35 & 36.158 & 37.626 \\
\hline 13 & $4.84 \mathrm{E}-05$ & $3.51 \mathrm{E}-05$ & $3.85 \mathrm{E}-05$ & $2.79 \mathrm{E}-05$ & 0.37 & 0.31 & 36.42133 & 37.189 \\
\hline 14 & $4.53 \mathrm{E}-05$ & $4.02 \mathrm{E}-05$ & $3.48 \mathrm{E}-05$ & $3.28 \mathrm{E}-05$ & 0.31 & 0.34 & 37.196 & 37.956 \\
\hline 15 & $5.41 \mathrm{E}-05$ & $4.85 \mathrm{E}-05$ & $3.36 \mathrm{E}-05$ & $3.02 \mathrm{E}-05$ & 0.36 & 0.36 & 36.787 & 37.476 \\
\hline 16 & $4.18 \mathrm{E}-05$ & $5.48 \mathrm{E}-05$ & $3.61 \mathrm{E}-05$ & $3.38 \mathrm{E}-05$ & 0.41 & 0.34 & 37.164 & 37.412 \\
\hline 17 & $4.86 \mathrm{E}-05$ & $5.06 \mathrm{E}-05$ & $3.26 \mathrm{E}-05$ & $3.15 \mathrm{E}-05$ & 0.35 & 0.32 & 37.164 & 36.968 \\
\hline 18 & $4.14 \mathrm{E}-05$ & $4.51 \mathrm{E}-05$ & $3.94 \mathrm{E}-05$ & $4.19 \mathrm{E}-05$ & 0.35 & 0.29 & 37.159 & 37.190 \\
\hline 19 & $4.38 \mathrm{E}-05$ & $5.23 \mathrm{E}-05$ & $3.41 \mathrm{E}-05$ & $3.74 \mathrm{E}-05$ & 0.36 & 0.38 & 36.597 & 37.207 \\
\hline 20 & $4.6 \mathrm{E}-05$ & $4.28 \mathrm{E}-05$ & $4.30 \mathrm{E}-05$ & $3.98 \mathrm{E}-05$ & 0.37 & 0.35 & 37.350 & 36.977 \\
\hline 21 & $4.22 \mathrm{E}-05$ & $4.53 \mathrm{E}-05$ & $3.87 \mathrm{E}-05$ & $2.63 \mathrm{E}-05$ & 0.37 & 0.31 & 37.392 & 38.036 \\
\hline 22 & $4.21 \mathrm{E}-05$ & $4.49 \mathrm{E}-05$ & $3.07 \mathrm{E}-05$ & $3.6 \mathrm{E}-05$ & 0.41 & 0.38 & 39.429 & 37.074 \\
\hline 23 & $4.6 \mathrm{E}-05$ & $5.36 \mathrm{E}-05$ & $3.75 \mathrm{E}-05$ & $4.05 \mathrm{E}-05$ & 0.34 & 0.31 & 37.151 & 37.769 \\
\hline 24 & $4.46 \mathrm{E}-05$ & $4.82 \mathrm{E}-05$ & $3.89 \mathrm{E}-05$ & $3.84 \mathrm{E}-05$ & 0.32 & 0.28 & 36.943 & 37.436 \\
\hline 25 & $4.11 \mathrm{E}-05$ & $5.11 \mathrm{E}-05$ & $3.80 \mathrm{E}-05$ & $4.48 \mathrm{E}-05$ & 0.37 & 0.34 & 37.255 & 38.352 \\
\hline 26 & $4.69 \mathrm{E}-05$ & $5.12 \mathrm{E}-05$ & $3.36 \mathrm{E}-05$ & $3.81 \mathrm{E}-05$ & 0.33 & 0.42 & 39.896 & 37.806 \\
\hline 27 & $4.93 \mathrm{E}-05$ & $5.22 \mathrm{E}-05$ & $3.63 \mathrm{E}-05$ & $4.14 \mathrm{E}-05$ & 0.37 & 0.32 & 38.376 & 37.567 \\
\hline 28 & $5.93 \mathrm{E}-05$ & $4.09 \mathrm{E}-05$ & $3.53 \mathrm{E}-05$ & $3.55 \mathrm{E}-05$ & 0.33 & 0.27 & 37.505 & 37.400 \\
\hline 29 & $3.91 \mathrm{E}-05$ & $4.03 \mathrm{E}-05$ & $3.29 \mathrm{E}-05$ & $2.62 \mathrm{E}-05$ & 0.32 & 0.36 & 37.812 & 38.072 \\
\hline 30 & $7.45 \mathrm{E}-05$ & $4.37 \mathrm{E}-05$ & $3.66 \mathrm{E}-05$ & $3.76 \mathrm{E}-05$ & 0.31 & 0.3 & 36.186 & 37.293 \\
\hline Min. & $3.91 \mathrm{E}-05$ & $3.51 \mathrm{E}-05$ & $3.08 \mathrm{E}-05$ & $2.62 \mathrm{E}-05$ & 0.24 & 0.27 & 36.158 & 36.968 \\
\hline Max. & $7.45 \mathrm{E}-05$ & $5.48 \mathrm{E}-05$ & $4.30 E-05$ & $4.5 \mathrm{E}-05$ & 0.44 & 0.42 & 43.261 & 38.874 \\
\hline Mean & $4.77 \mathrm{E}-05$ & $4.62 \mathrm{E}-05$ & $3.59 \mathrm{E}-05$ & $3.57 \mathrm{E}-05$ & 0.35 & 0.33 & 37.730 & 37.614 \\
\hline Std. dev. & $7.63 \mathrm{E}-06$ & $4.9 \mathrm{E}-06$ & $2.93 \mathrm{E}-06$ & $5.45 \mathrm{E}-06$ & 0.04 & 0.03 & 1.608 & 0.491 \\
\hline
\end{tabular}

hypothesis can be rejected. There is a significant difference between two approaches, and random immigrants MOPSO outperforms mutation MOPSO algorithm.
Table 9 Statistical hypothesis testing for the fourth test problem

\begin{tabular}{lll}
\hline Performance metric & $z$ value & $H_{0}$ \\
\hline $\mathrm{S}$ & 0.9411 & Not enough evidence to reject \\
$\mathrm{D}$ & 0.1451 & Not enough evidence to reject \\
$\mathrm{E}$ & 2.2727 & Reject \\
$\mathrm{T}$ & 0.3786 & Not enough evidence to reject \\
\hline$\alpha<0.05$ & &
\end{tabular}




\section{Results for the third test problem}

According to the results given in Tables 6 and 7, null hypothesis cannot be rejected. It means that there is no significant difference between two approaches with respect to these performance metrics. Regarding the time performance metric, null hypothesis can be rejected, and the mutation MOPSO outperforms the random immigrants MOPSO.

\section{Results for the fourth test problem}

According to the results given in Tables 8 and 9, for $S$ and $D$ performance metrics, null hypothesis cannot be rejected. It means that there is no significant difference between two approaches with respect to these performance metrics. Nevertheless, for $E$ performance metric, null hypothesis can be rejected. It means that there is a significant difference between two approaches with respect to $E$ performance metric, and mutation MOPSO approach outperforms random immigrants MOPSO approach. Regarding the time performance metric, null hypothesis cannot be rejected. In other words, there is no significant difference between two approaches with respect to the running times.

\section{Conclusion}

Computational Intelligence is developed to create solutions for the real-life complex problems for which linear, nonlinear, or stochastic models could not propose a remedy. Nature-inspired metaheuristic algorithms take an improving importance in computational intelligence. The most important issue in metaheuristics is to balance the exploitation and the exploration depending on the problem studied. When the problem has a single objective, the hybridized algorithms provide solutions, but it is more difficult when a multi objective analysis is made.

This paper is original to develop MOPSO using Random Immigrants, which has enlarged the diversity of solutions remarkably. Application of the proposed algorithm on four well-accepted data sets has shown the benefits of the solution for four performance measures, process timing, Generational Distance, Spacing, and Error Ratio. Statistical experiments are constructed to compare the Mutation Approach (that gives better than other existing solutions) and the Random Immigrants approach for diversity. Results show that Random Immigrants approach is faster in providing the solution in most of the cases and as good as mutation approach in the others.
Implementing the proposed algorithm in a real-life case and to compare the results (for both real-life cases and test suits) with the state-of-the-art variants of the MOPSO will be the extension of this study. Archive maintenance and local optima problems faced in using MOPSO worth new studies, which will be further analysis in our team. Having observed the timing performances of MOPSO with Random Immigrants, we would also like to recommend the application of this method in Big Data handling.

This paper will open a new dimension for the MOPSO researchers and provide a new tool for computational intelligence application.

Open Access This article is licensed under a Creative Commons Attribution 4.0 International License, which permits use, sharing, adaptation, distribution and reproduction in any medium or format, as long as you give appropriate credit to the original author(s) and the source, provide a link to the Creative Commons licence, and indicate if changes were made. The images or other third party material in this article are included in the article's Creative Commons licence, unless indicated otherwise in a credit line to the material. If material is not included in the article's Creative Commons licence and your intended use is not permitted by statutory regulation or exceeds the permitted use, you will need to obtain permission directly from the copyright holder. To view a copy of this licence, visit http://creativecomm ons.org/licenses/by/4.0/.

\section{References}

1. Agrawal S, Dashora Y, Tiwari MK, Son YJ (2008) Interactive particle swarm: a pareto-adaptive metaheuristic to multiobjective optimization. IEEE Trans Syst Man Cybern Part A Syst Hum 38(2):258-277

2. Al Moubayed N, Petrovski A, McCall J (2010) A novel smart multi-objective particle swarm optimisation using decomposition. Springer, Berlin Heidelberg, pp 1-10

3. Baltar AM, Fontane DG (2006) A generalized multiobjective particle swarm optimization solver for spreadsheet models: application to water quality. Hydrol Days 1-12

4. Coello CAC, Lechuga MS (2002) Mopso: a proposal for multiple objective particle swarm optimization. In: Proceedings of the 2002 congress on evolutionary computation, 2002. CEC '02, vol 2, pp 1051-1056

5. Coello CAC, Pulido GT, Lechuga MS (2004) Handling multiple objectives with particle swarm optimization. IEEE Trans Evol Comput 8(3):256-279

6. Coello Coello CA, González Brambila S, Figueroa Gamboa J, Castillo Tapia MG, Hernández Gómez R (2019) Evolutionary multiobjective optimization: open research areas and some challenges lying ahead. Complex Intell Syst. https://doi.org/10.1007/s40747019-0113-4

7. Daneshyari M, Yen GG (2011) Cultural-based multiobjective particle swarm optimization. IEEE Trans Syst Man Cybern Part B (Cybernetics) 41(2):553-567

8. Deb K (1999) Multi-objective genetic algorithms:problem difficulties and construction of test problems. Evol Comput 7:205-230 
9. Fan SKS, Chang JM, Chuang YC (2015) A new multi-objective particle swarm optimizer using empirical movement and diversified search strategies. Eng Optim 47(6):750-770

10. Fieldsend JE, Singh S (2002) A multi-objective algorithm based upon particle swarm optimisation, an efficient data structure and turbulence. In: 2002 UK workshop on computational intelligence, Birmingham, UK, 2-4 September 2002, pp. 37-44

11. Han H, Lu W, Zhang L, Qiao J (2018) Adaptive gradient multiobjective particle swarm optimization. IEEE Trans Cybern 48(11):3067-3079. https://doi.org/10.1109/TCYB.2017.2756874

12. Hu W, Yen GG (2013) Density estimation for selecting leaders and mantaining archive in mopso. In: 2013 IEEE congress on evolutionary computation, pp 181-188

13. Hu W, Yen GG (2015) Adaptive multiobjective particle swarm optimization based on parallel cell coordinate system. IEEE Trans Evol Comput 19(1):1-18

14. Izui K, Nishiwaki S, Yoshimura M, Nakamura M, Renaud JE (2008) Enhanced multiobjective particle swarm optimization in combination with adaptive weighted gradient-based searching. Eng Optim 40(9):789-804

15. Kennedy J, Eberhart R (1995) Particle swarm optimization. In: IEEE international conference on neural networks, 1995. Proceedings, vol 4, pp 1942-1948

16. Kita H, Yabumoto Y, Mori N, Nishikawa Y (1996) Multi-objective optimization by means of the thermodynamical genetic algorithm. In: Proceedings of the 4 th international conference on parallel problem solving from nature, PPSN IV. Springer, London, pp 504-512

17. Konak A, Coit DW, Smith AE (2006) Multi-objective optimization using genetic algorithms: a tutorial. Reliab Eng Syst Saf 91(9):9921007. Special Issue-Genetic Algorithms and ReliabilitySpecial Issue-Genetic Algorithms and Reliability

18. Kursawe F (1991) A variant of evolution strategies for vector optimization. In: Proceedings of the 1st workshop on parallel problem solving from nature, PPSN I. Springer, London, pp 193-197

19. Lalwani S, Singhal S, Kumar R, Gupta N (2013) A comprehensive survey: applications of multi-objective particle swarm optimization (mopso) algorithm. Trans Combin 2(1):39-101

20. Leung MF, Ng SC, Cheung CC, Lui AK (2014) A new strategy for finding good local guides in mopso. In: 2014 IEEE congress on evolutionary computation (CEC), pp 1990-1997

21. Li X (2004) Better spread and convergence: particle swarm multiobjective optimization using the maximin fitness function. Springer, Berlin Heidelberg, pp 117-128

22. Luo J, Huang X, Li X, Gao K (2019) A novel particle swarm optimizer for many-objective optimization. In: 2019 IEEE congress on evolutionary computation (CEC), pp 958-965. https://doi.org/ 10.1109/CEC.2019.8790343

23. Mahmoodabadi MJ, Bagheri A, Nariman-zadeh N, Jamali A (2012) A new optimization algorithm based on a combination of particle swarm optimization, convergence and divergence operators for single-objective and multi-objective problems. Eng Optim 44(10):1167-1186

24. Markowitz H (1952) Portfolio selection. J Fin 7(1):77-91

25. Mavrovouniotis M, Yang S (2013) Ant colony optimization with immigrants schemes for the dynamic travelling salesman problem with traffic factors. Appl Soft Comput 13(10):4023-4037

26. Meza J, Espitia H, Montenegro C, Giménez E, González-Crespo R (2017) Movpso: Vortex multi-objective particle swarm optimization. Appl Soft Comput 52:1042-1057. https://doi.org/10.1016/j. asoc.2016.09.026

27. Moore J, Chapman R, Dozier G (2000) Multiobjective particle swarm optimization. In: Proceedings of the 38th annual on southeast regional conference, ACM-SE 38. ACM, New York, pp 56-57

28. Mostaghim S, Teich J (2003) Strategies for finding good local guides in multi-objective particle swarm optimization (mopso). In:
Swarm intelligence symposium, 2003. SIS '03. Proceedings of the 2003 IEEE, pp 26-33

29. Padhye N (2009) Comparison of archiving methods in multiobjectiveparticle swarm optimization (mopso): Empirical study. In: Proceedings of the 11th annual conference on genetic and evolutionary computation, GECCO '09. ACM, New York, pp 1755-1756

30. Padhye N, Branke J, Mostaghim S (2009) Empirical comparison of mopso methods: guide selection and diversity preservation. In: 2009 IEEE congress on evolutionary computation, pp 2516-2523

31. Pan A, Wang L, Guo W, Wu Q (2018) A diversity enhanced multiobjective particle swarm optimization. Inf Sci 436-437:441-465. https://doi.org/10.1016/j.ins.2018.01.038

32. Peng W, Zhang Q (2008) A decomposition-based multi-objective particle swarm optimization algorithm for continuous optimization problems. In: 2008 IEEE international conference on granular computing, pp 534-537

33. Pulido GT, Coello Coello CA (2004) Using clustering techniques to improve the performance of a multi-objective particle swarm optimizer. Springer, Berlin, pp 225-237

34. Raquel CR, Naval Jr PC (2005) An effective use of crowding distance in multiobjective particle swarm optimization. In: Proceedings of the 7 th annual conference on genetic and evolutionary computation, GECCO '05. ACM, New York, pp 257-264

35. Scheepers C, Engelbrecht AP (2017) Vector evaluated particle swarm optimization: The archive's influence on performance. In: 2017 IEEE congress on evolutionary computation (CEC), pp 565572. https://doi.org/10.1109/CEC.2017.7969361

36. Sierra MR, Coello CAC (2006) Multi-objective particle swarm optimizers: a survey of the state-of-the-art. Int J Comput Intell Res 2(3):287-308

37. Sierra MR, Coello Coello CA (2005) Improving pso-based multiobjective optimization using crowding, mutation and $\epsilon$-dominance. In: Proceedings of the third international conference on evolutionary multi-criterion optimization, EMO'05. Springer, Berlin, Heidelberg, pp 505-519

38. Unal AN (2013) A Genetic Algorithm for the Multiple Knapsack Problem in Dynamic Environment. In: World congress on engineering and computer science, WCECS 2013, vol II, Lecture Notes in Engineering and Computer Science, pp 1162-1167

39. Url-1: http://delta.cs.cinvestav.mx/ ccoello/EMOO/testfuncs/ (2016). Accessed 26 July 2016

40. Url-2: http://people.brunel.ac.uk/ mastjjb/jeb/orlib/portinfo.html (2016). Accessed 26 July 2016

41. Xiang Y, Zhou Y, Chen Z, Zhang J (2018) A many-objective particle swarm optimizer with leaders selected from historical solutions by using scalar projections. IEEE Trans Cybern 1-14. https://doi.org/ 10.1109/TCYB.2018.2884083

42. Xiao-hua Z, Hong-yun M, Li-cheng J (2005) Intelligent particle swarm optimization in multiobjective optimization. In: 2005 IEEE congress on evolutionary computation, vol 1, pp 714-719

43. Yang S (2005) Memory-based immigrants for genetic algorithms in dynamic environments. In: Proceedings of the 7th annual conference on genetic and evolutionary computation, GECCO '05, pp 1115-1122. ACM, New York, NY, USA

44. Yen GG, Leong WF (2009) Dynamic multiple swarms in multiobjective particle swarm optimization. IEEE Trans Syst Man Cybern Part A Syst Hum 39(4):890-911

45. Yen GG (2010) Leong, W.F.: Constraint handling procedure for multiobjective particle swarm optimization. In: IEEE congress on evolutionary computation, pp 1-8

46. Zapotecas Martínez S, Coello Coello CA (2011) A multi-objective particle swarm optimizer based on decomposition. In: Proceedings of the 13th annual conference on genetic and evolutionary computation, GECCO '11. ACM, New York, pp 69-76. DOI https://doi. org/10.1145/2001576.2001587 
47. Zhang H, Sun J, Liu T, Zhang K, Zhang Q (2019) Balancing exploration and exploitation in multiobjective evolutionary optimization. Inf Sci 497:129-148. https://doi.org/10.1016/j.ins.2019.05.046

48. Zhao SZ, Suganthan PN (2011) Two-lbests based multi-objective particle swarm optimizer. Eng Optim 43(1):1-17

49. Zhu Q, Lin Q, Chen W, Wong K, Coello Coello CA, Li J, Chen J, Zhang J (2017) An external archive-guided multiobjective particle swarm optimization algorithm. IEEE Trans Cybern 47(9):2794 2808. https://doi.org/10.1109/TCYB.2017.2710133
50. Zitzler E, Deb K, Thiele L (2000) Comparison of multiobjective evolutionary algorithms: empirical results. Evol Comput 8(2):173195

Publisher's Note Springer Nature remains neutral with regard to jurisdictional claims in published maps and institutional affiliations. 